\title{
Balanced Circle Packings for Planar Graphs
}

\author{
Md. Jawaherul Alam ${ }^{1}$, David Eppstein ${ }^{2}$, Michael T. Goodrich ${ }^{2}$, \\ Stephen G. Kobourov ${ }^{1}$, and Sergey Pupyrev ${ }^{1,3}$ \\ ${ }^{1}$ Department of Computer Science, University of Arizona, Tucson, Arizona, USA \\ 2 Department of Computer Science, University of California, Irvine, California, USA \\ ${ }^{3}$ Institute of Mathematics and Computer Science, Ural Federal University, Russia
}

\begin{abstract}
We study balanced circle packings and circle-contact representations for planar graphs, where the ratio of the largest circle's diameter to the smallest circle's diameter is polynomial in the number of circles. We provide a number of positive and negative results for the existence of such balanced configurations.
\end{abstract}

\section{Introduction}

Circle packings are a frequently used and important tool in graph drawing [3, 5, 10, 11 , [18]. In this application, they can be formalized using the notion of a circle-contact representation for a planar graph; this is a collection of interior-disjoint circles in $\mathbb{R}^{2}$, corresponding one-for-one with the vertices of the graph, such that two vertices are adjacent if and only if their corresponding two circles are tangent to each other [15]. In a classic paper, Koebe [16] proved that every triangulated planar graph has a circle-contact representation, and this has been subsequently re-proved several times. Generalizing this, every planar graph has a circle-contact representation: we can triangulate the graph by adding "dummy" vertices connected to the existing vertices within each face, produce a circle-contact representation for this augmented graph, and then remove the circles corresponding to dummy vertices. It is not always possible to describe a circle-contact representation for a given graph by a symbolic formula involving radicals [2, 5], but they can nevertheless be constructed numerically and efficiently by polynomial-time iterative schemes [7, 19].

One of the drawbacks of some of these constructions, however, is that the sizes of the circles in some of these configurations may vary exponentially, leading to drawings with very high area or with portions that are so small that they are below the resolution of the display. For this reason, we are interested in balanced circle packings and circle-contact representations for planar graphs, where the ratio of the maximum and minimum diameters for the set of circles is polynomial in the number of vertices in the graph; see Fig. 1

Related Work. There is a large body of work about representing planar graphs as contact graphs, where vertices are represented by geometrical objects and edges correspond to two objects touching in some pre-specified fashion. For example, Hliněný [15] studies contact representations using curves and line segments as objects. Several authors have considered contact graphs of triangles of various types. For instance, de Fraysseix et al. [12] show that every planar graph has a triangle-contact representation, and

C. Duncan and A. Symvonis (Eds.): GD 2014, LNCS 8871, pp. 125136 2014.

(c) Springer-Verlag Berlin Heidelberg 2014 


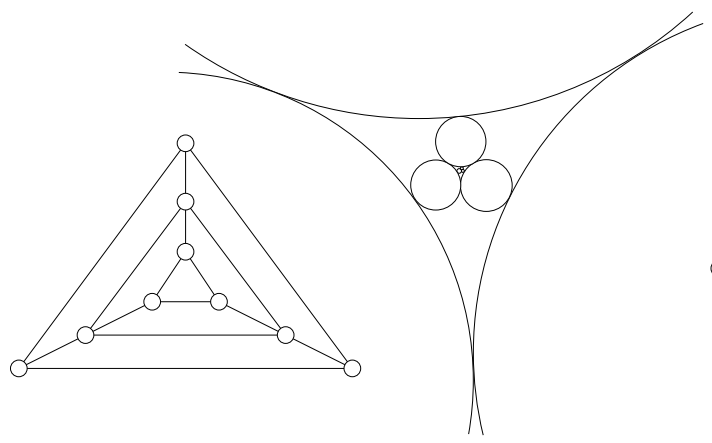

(a)

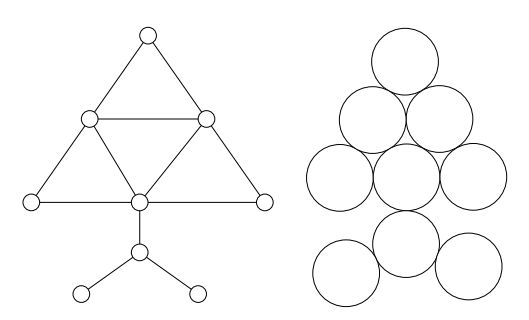

(b)

Fig. 1. Two planar graphs with possible circle-contact representations: (a) a representation that is not optimally balanced; (b) a perfectly-balanced representation

Gonçalves et al. [14] prove that every 3-connected planar graph and its dual can be simultaneously represented by touching triangles (and they point out that 4-connected planar graphs also have contact representations with homothetic triangles). Also, Duncan et al. [9] show that every planar graph has a contact representation with convex hexagons all of whose sides have one of three possible slopes, and that hexagons are necessary for some graphs, if convexity is required. With respect to balanced circlecontact representations, Breu and Kirkpatrick [4] show that it is NP-complete to test whether a graph has a perfectly-balanced circle-contact representation, in which every circle is the same size.

New Results. In this paper, we provide a number of positive and negative results regarding balanced circle-contact representations for planar graphs:

- Every planar graph with bounded maximum vertex degree and logarithmic outerplanarity admits a balanced circle-contact representation.

- There exist planar graphs with bounded maximum degree and linear outerplanarity, or with linear maximum degree and bounded outerplanarity, that do not admit a balanced circle-contact representation.

- Every tree admits a balanced circle-contact representation.

- Every outerpath admits a balanced circle-contact representation.

- Every cactus graph admits a balanced circle-contact representation.

- Every planar graph with bounded tree-depth admits a balanced circle-contact representation.

\section{Bounded Degree and Logarithmic Outerplanarity}

A plane graph (that is, a combinatorially fixed planar embedding of a planar graph) is outerplanar if all of its vertices are on the outer face. A $k$-outerplanar graph is defined recursively. As a base case, if a plane graph is outerplanar, then it is a 1-outerplanar graph. A plane graph is $k$-outerplanar, for $k>1$, if the removal of all the outer vertices 
(and their incident edges) yields a graph such that each of the remaining components is $(k-1)$-outerplanar. The outerplanarity of a plane graph $G$ is the minimum value for $k$ such that $G$ is $k$-outerplanar.

\subsection{Balanced Circle-Contact Representations}

Theorem 1. Every $n$-vertex $k$-outerplanar graph with maximum degree $\Delta$ admits a circle-contact representation where the ratio of the maximum and the minimum diameter is at most $f(\Delta)^{k+\log n}$, for some positive function $f$. In particular, when $\Delta$ is a fixed constant and $k$ is $\mathcal{O}(\log n)$, this ratio is polynomial in $n$.

In order to prove the theorem, we need the following result from [18].

Lemma 1 (Malitz-Papakostas). The vertices of every triangulated planar graph $G$ with the maximum degree $\Delta$ can be represented by nonoverlapping disks in the plane so that two disks are tangent to each other if and only if the corresponding vertices are adjacent, and for each two disks that are tangent to each other, the ratio of the radii of the smaller to the larger disk is at least $\alpha^{\Delta-2}$ with $\alpha=\frac{1}{3+2 \sqrt{3}} \approx 0.15$.

As a direct corollary, every maximal planar graph with maximum degree $\Delta=\mathcal{O}(1)$ and diameter $d=\mathcal{O}(\log n)$ has a balanced circle-contact representation. Theorem 1 goes beyond this.

Proof of Theorem 1; To prove the claim, it is sufficient to show how to augment a given $k$-outerplanar graph into a maximal planar graph with additional vertices so that its maximum degree remains $\mathcal{O}(\Delta)$ and its diameter becomes $\mathcal{O}(k+\log n)$. By Lemma 1 , the resulting graph admits a circular contact representation with the given bounds on the ratio of radii. Removing the circles corresponding to the added vertices yields the desired balanced representation of the original graph.

Let $G$ be an $n$-vertex $k$-outerplanar graph with the maximum degree $\Delta$. If the outerplanarity $k$ of $G$ is bounded by a constant, we can easily augment $G$ to logarithmic diameter, preserving its constant maximum degree, as follows. Inside each non-triangular face $f$ of $G$, insert a balanced binary tree with $\lceil\log |f|\rceil$ levels and $|f|$ leaves and then triangulate the remaining non-triangular faces by inserting an outerpath (an outerplanar graph whose weak dual is a path) with constant maximum degree; see Fig. 2 . However, such an augmentation results in a maximal planar graph with the diameter

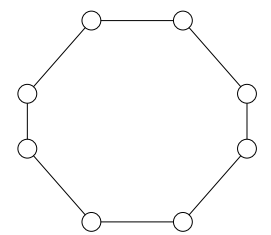

(a)

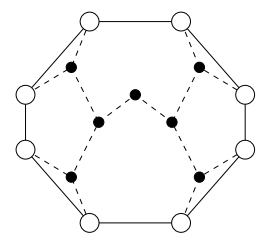

(b)

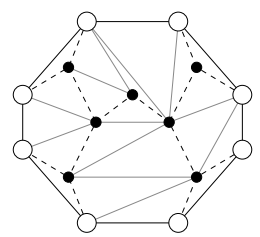

(c)

Fig. 2. (a) A face, (b) augmentation with a balanced binary tree, (c) triangulation with grey edges 
$d=\mathcal{O}(k \log n)$, which does not yield a balanced circle-contact representation when $k$ is non-constant. For $k=\Omega(\log n)$, we present a different augmentation to achieve the diameter $d=\mathcal{O}(k+\log n)$ in the resulting graph.

We augment the graph using weight-balanced binary trees. Let $T$ be a binary tree with leaves $l_{1}, l_{2}, \ldots, l_{|f|}$ and a prespecified weight $w_{i}$ assigned to each leaf $l_{i}$. The tree $T$ is weight-balanced if the depth of each leaf $l_{i}$ in $T$ is $\mathcal{O}\left(\left\lceil\log \left(W / w_{i}\right)\right\rceil\right)$, where $W=\sum_{i=1}^{f} w_{i}$. There exist several algorithms for producing a weight-balanced binary tree with positive integer weights defined on its leaves [13,21].

To augment $G$, we label each vertex $v$ of $G$ with the number $l+1$, where $l$ is the number of outer cycles that need to be removed before $v$ becomes an outer vertex. By our assumption that the outerplanarity of $G$ is $k$, the label of every vertex is at most $k$. It follows from this labeling that, for each vertex $v$ of $G$ with label $l>1$, there exists a face $f$ containing $v$ such that $f$ has at least one vertex of label $l-1$ and such that all the vertices on $f$ have label either $l$ or $l-1$. We insert a weight-balanced binary tree inside $f$; we choose an arbitrary vertex of $f$ with label $l-1$ as the root of the tree, and a subset of vertices with label $l$ as the leaves; see Fig. 3. We construct these trees inside the different faces in such a way that each vertex of $G$ with label $l>1$ becomes a leaf in exactly one of the trees. Finally, we insert another weight-balanced tree $T_{0}$ on the outer face containing all the outer vertices as the leaves. Note that we have yet to specify the weights we assign to these leaves for producing the weight-balanced trees. By the construction, the union of all these trees forms a connected spanning tree of $G$; we can consider the root of $T_{0}$ to be the root of the whole spanning tree.

Let us now specify the weights assigned to the leaves of the different weight-balanced trees. We label each tree with the label of its root, and define the weights for the leaves of each tree in a bottom-up ordering, by decreasing order of the labels of the trees. In a tree $T$ with label $l=(k-1)$, all the leaves have label $k$ and are not the root of any other tree; we assign each of these leave the weight 1 . In this case, the total weight of $T$ is the number of its leaves. Similarly, for a tree with label $l<k-1$, we assign a weight

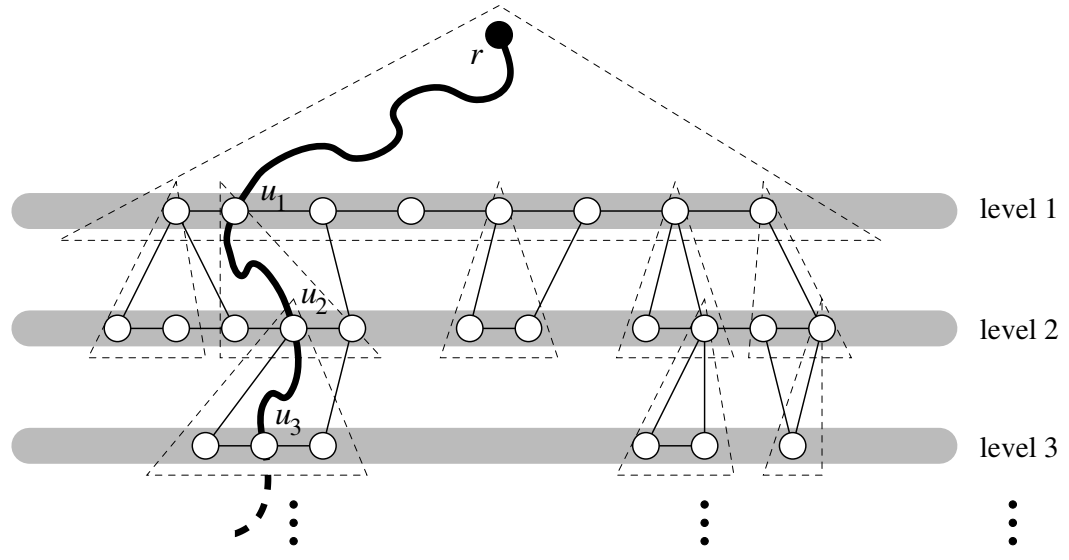

Fig. 3. Augmentation of $G$ with a weight-balanced binary trees 


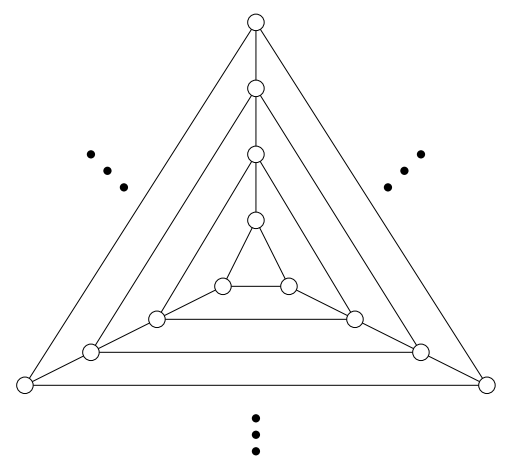

(a)

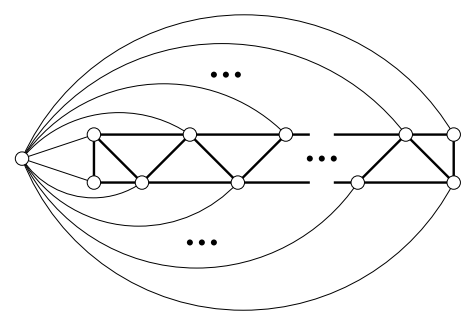

(b)

Fig. 4. Planar graphs with no balanced circle-contact representation: (a) the nested-triangles graph [8]; (b) a 2-outerplanar graph

of 1 to those leaves $v$ that do not have any tree rooted at them; otherwise, if $v$ is the root of a tree $T_{v}$ with label $l+1$, the weight of $v$ is the total weight of $T_{v}$. The total weight of $T$ is defined as the summation of the weights of all its leaves.

Now, for each vertex $v$ of $G$, the distance to $v$ from the root $r$ of $T_{0}$ is $\mathcal{O}(k+\log n)$. Indeed, assume that $v=u_{l}$ is a vertex with label $l$ and $u_{l-1}, \ldots, u_{1}, u_{0}=r$ are the root vertices of the successive weight-balanced trees $T_{u_{l-1}}, \ldots, T_{u_{1}}, T_{0}$ with labels $l-$ $1, \ldots, 1,0$, respectively on the way from $v$ to $r$; see Fig. 3. Then the distance from $v$ to $r$ is $\mathcal{O}\left(\left\lceil\log w(r) / w\left(u_{1}\right)\right\rceil\right)+\mathcal{O}\left(\left\lceil\log w\left(u_{1}\right) / w\left(u_{2}\right)\right\rceil\right)+\ldots+\mathcal{O}\left(\left\lceil\log w\left(u_{l-1}\right) / w(v)\right\rceil\right)=$ $\mathcal{O}(k+\log w(r))$. Here $w\left(u_{i}\right)$ denotes the weight of vertex $u_{i}$ as the root; $w(r)$ is the weight of the root of $T_{0}$, which is equal to the total number of vertices, $n$, in $G$. Therefore, the diameter of the augmented graph is $\mathcal{O}(k+\log n)$, where the first term, $k$, comes from the ceilings in the summation. Finally, we triangulate the graph by inserting outerpaths with constant maximum degree inside each non-triangular face to obtain a maximal planar graph with constant maximum degree and $\mathcal{O}(k+\log n)$ diameter. The result follows from Lemma 1 .

\subsection{Negative Results}

Next we show that, for a graph with unbounded maximum degree or unbounded outerplanarity, there might not be a balanced circle-contact representation with circles.

Lemma 2. There is no balanced circle-contact representation for the graphs in Fig. 4

Lemma 2, which we prove in the full version of this paper [1], shows the tightness of the two conditions for balanced circle-contact representations in Theorem 1. Note that the example of the graph in Fig. 4(b) can be extended for any specified maximum degree, by adding a simple path to the high-degree vertex. Furthermore, the example is a 2-outerplanar graph with no balanced circle-contact representation. 


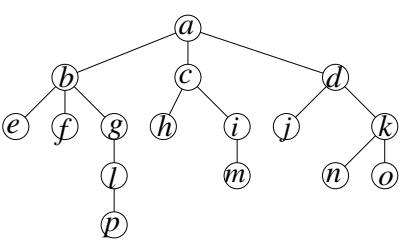

(a)

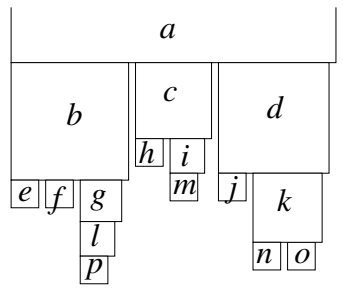

(b)

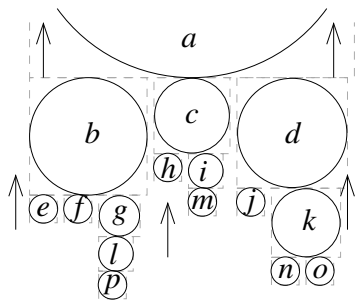

(c)

Fig. 5. Construction of a balanced circle-contact representation

\section{Trees and Outerplanar Graphs}

Theorem 2. Every tree has a balanced circle-contact representation. Such a representation can be found in linear time.

Proof: We first find a contact representation $\Gamma$ of a given tree $T$ with squares such that the ratio of the maximum and the minimum sizes for the squares is polynomial in the number of vertices $n$ in $T$. To this end, we consider $T$ as a rooted tree with an arbitrary vertex $r$ as the root. Then we construct a contact representation of $T$ with squares where each vertex $v$ of $T$ is represented by a square $R(v)$ such that $R(v)$ touches the square for its parent by its top side and it touches all the squares for its children by its bottom side; see Figs. 5(a) and 5(b) We choose the size of $R(v)$ as $l(v)+\varepsilon(n(v)-1)$, where $\varepsilon>0$ is a small positive constant and $n(v)$ and $l(v)$ denote the number of vertices and the number of leaves in the subtree of $T$ rooted at $v$. In particular, the size of $R(v)$ is 1 when $v$ is a leaf. If $v$ is not a leaf, then suppose $v_{1}, \ldots, v_{d}$ are the children of $v$ in the counterclockwise order around $v$. Then we place the squares $R\left(v_{1}\right), \ldots, R\left(v_{d}\right)$ from left-to-right touching the bottom side of $R(v)$ such that for each $i \in\{1, \ldots, d-1\}$, $R\left(v_{i+1}\right)$ is placed $\varepsilon$ unit to the right of $R\left(v_{i}\right)$; see Fig. 5(b). There is sufficient space to place all these squares in the bottom side of $R(v)$, since $n(v)=\left(\sum_{i=1}^{d} n\left(v_{i}\right)\right)-1$ and $l(v)=\sum_{i=1}^{d} l\left(v_{i}\right)$. The representation contains no crossings or unwanted contacts since for each vertex $v$, the representation of the subtrees rooted at $v$ is bounded in the left and right side by the two sides of $R(v)$, and all the subtrees rooted at the children of $v$ are in disjoint regions $\varepsilon$ unit away from each other. The size of the smallest square is 1 , while the size of the largest square (for the root) has size $l(T)+\varepsilon(n-1)=\mathcal{O}(n)$, where $l(T)$ is the number of leaves in $T$.

Using $\Gamma$, we find a balanced circle-contact representation of $T$ as follows. We replace each square $R(v)$, representing vertex $v$, by an inscribed circle of $R(v)$; see Fig. 5(c), The operation removes some contacts from the representation. We re-create these contacts by a top-down traversal of $T$ and moving each circle upward until it touches its parent. Note that a given circle will not touch or intersect any circle other than the circles for its parent and its children, as for every vertex in the infinite strip between its leftmost and rightmost point for its circle, the closest circle in the upward direction is 
its parent's one. Thus, we obtain a contact representation of $T$ with circles. The representation is balanced since the diameter for every circle is equal to the side-length for its square and we started with a balanced representation $\Gamma$.

The linear running time can be achieved by a linear-time traversal of $T$. First, by a bottom-up traversal of $T$, we compute the values $n(v)$ and $l(v)$ for each vertex $v$ of $T$. Using the values for each vertex, we compute the square-contact representation for $T$ by a linear-time top-down traversal of $T$. Finally, in another top-down traversal of $T$, for each vertex $v$ of $T$, we can compute the exact translation required for the inscribed circles of $R(v)$ to touch the parent circle.

Let us now describe how to compute a balanced circle-contact representation for a cactus graph, which is a connected graph in which every biconnected component is either an edge or a cycle. We use the algorithm described in the proof of Theorem 2 , and we call it Draw_Tree.

Let $T$ be a rooted tree with a plane embedding. For each vertex $v$ of $T$, add an edge between every pair of the children of $v$ that are consecutive in the clockwise order around $v$. Call the resulting graph an augmented fan-tree for $T$. Clearly for any rooted tree $T$, the augmented fan-tree is outerplanar. We call an outerplanar graph a fan-tree graph if it is an augmented fan-tree for some rooted tree. A star is the complete bipartite graph $K_{1, n-1}$. The center of a star is the vertex that is adjacent to every other vertex. An augmented fan-tree for a star is obtained by taking the center as the root. Thus, an augmented fan-tree for a star is a fan. The center of a fan is again the vertex adjacent to all the other vertices.

Lemma 3. Every subgraph of a fan admits a contact representation with circles in which, for each circle $c(v)$ representing a vertex $v$ other than the center, the vertical strip containing $c(v)$ is empty above $c(v)$.

Proof: Let $G$ be a subgraph of a fan and let $T$ be the star contained in the fan. We now use the contact representation $\Gamma$ of $T$ obtained by Draw_Tree to compute a representation for $G$. Consider the square-contact representation computed for $T$ in the algorithm. This defines a vertical strip for each circle $c(v)$ in $\Gamma$ representing a vertex $v$, and for all the vertices other than the center, these strips are disjoint; see Fig. 6(a). Call the left and right boundary of this strip the left- and right-line for $c$, respectively.

We now consider a set $S$ of circles, one for each vertex of $G$ other than the center, with the following properties:

(P1) The circles are interior-disjoint.

(P2) Each circle $c^{\prime}(v)$ representing a vertex $v$ spans the entire width of the vertical strip for $v$, and the vertical strip above $c^{\prime}(v)$ is empty.

(P3) For each vertex $v$, the circle $c^{\prime}(v)$ touches the circle $c_{0}$ representing the center in $\Gamma$ if $v$ is adjacent to the center; otherwise, $c^{\prime}(v)$ is exactly $\varepsilon$ distance away from $c_{0}$, for some fixed constant $\varepsilon>0$.

(P4) If a vertex $v$ is not adjacent to the vertex on its left (or if $v$ is the leftmost vertex), then the leftmost point of $c^{\prime}(v)$ is on the left-line of $v$; similarly, if $v$ is not adjacent to the vertex on its right (or if $v$ is the rightmost vertex), then the rightmost point of $c^{\prime}(v)$ is on the right-line of $v$. 

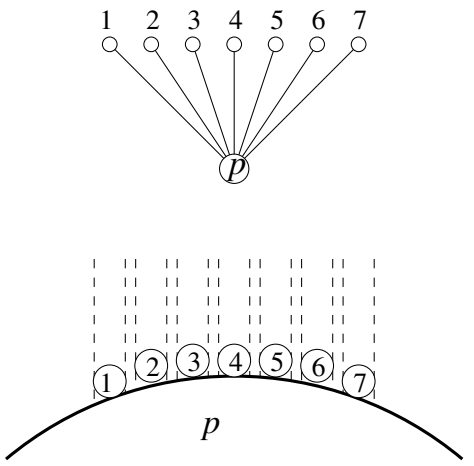

(a)
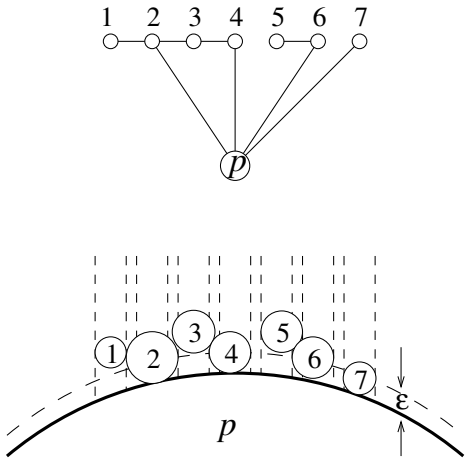

(b)

Fig. 6. (a) A star $T$ and a contact representation of $T$ with circles; (b) a subgraph of the fan for $T$ and its contact representation with circles

(P5) The sizes for the circles are maximal with respect to the above properties.

Note that there exists a set of circles with the properties (P1)-(P4); in particular, the set of circles in $\Gamma$ representing the vertices of $T$ other than the center is such a set. We now claim that the set $S$ of circles with properties (P1)-(P5) together with the circle $c_{0}$ gives a contact representation for $G$; see Fig. 6(b). First note that a circle $c^{\prime}(v)$ cannot touch any circle other than $c_{0}$ and the two circles $c\left(v_{l}\right)$ and $c\left(v_{r}\right)$ representing the vertices $v_{l}$ and $v_{r}$ on its left and right, respectively. Indeed, it cannot pass the vertical strip for $v_{l}$ and $v_{r}$ above them due to (P2) and behind them due to (P3). Furthermore, the $\varepsilon$ distance between $c_{0}$ and the circles for vertices non-adjacent to the center and the restriction on the left and right side in (P4) ensures that there is no extra contact. Hence, it is sufficient to show that for each edge in $G$, we have the contact between the corresponding circles.

Since each circle $c^{\prime}(v)$ is maximal in size, it must touch at least three objects. One of them is either the circle $c_{0}$ or the $\varepsilon$ offset line for $c_{0}$. Thus, if $v_{l}$ and $v_{r}$ are the left and right neighbors of $v$ (if any), then $c^{\prime}(v)$ must touch two of the followings: (i) $c^{\prime}\left(v_{l}\right)$ (or the left line of $v$ if $v_{l}$ does not exists), (ii) the right line for $v_{l}$, (iii) $c^{\prime}\left(v_{r}\right)$ (or the right line of $v$ if $v_{r}$ does not exists), and (iv) the left line for $v_{r}$. Assume without loss of generality that both $v_{l}$ and $v_{r}$ exist for $v$. Then if $c^{\prime}(v)$ touches both $c^{\prime}\left(v_{l}\right)$ and $c^{\prime}\left(v_{r}\right)$, we have the desired contacts for $v$. Therefore, for a desired contact of $c^{\prime}(v)$ to be absent, either $c^{\prime}(v)$ touches both $c^{\prime}\left(v_{l}\right)$ and the right-line of $v_{l}$ (and misses the contact with $c^{\prime}\left(v_{r}\right)$ ), or it touches both $c^{\prime}\left(v_{r}\right)$ and the left-line of $v_{r}$ (and misses the contact with $c^{\prime}\left(v_{r}\right)$ ).

Assume, for the sake of a contradiction, that there are two consecutive vertices $x$ and $y$ that are adjacent in $G$ but $c^{\prime}(x)$ and $c^{\prime}(y)$ do not touch each other. Let $l$ and $r$ be the vertices to the left of $x$ and to the right of $y$, respectively. Then it must be the case that $x$ touches both $c^{\prime}(l)$ and the right line for $l$ and $y$ touches both $c^{\prime}(r)$ and the left line of $r$; see Fig. 7(a), One can then increase the size of either $c^{\prime}(x)$ or $c^{\prime}(y)$ (say $c^{\prime}(y)$ ) such 


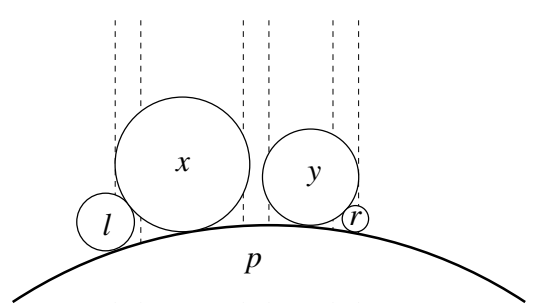

(a)

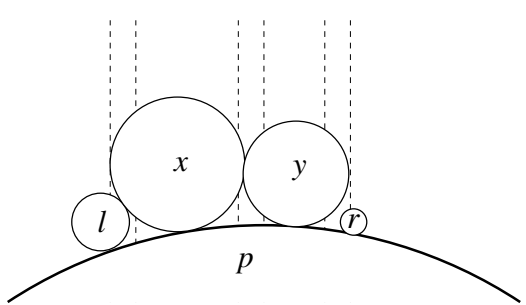

(b)

Fig. 7. Illustration for the proof of Lemma 3 if the circles for $x$ and $y$ do not touch each other, at least one can be increased in size

that it now touches $c^{\prime}(x)$ and the left-line for $r$ (but not $c^{\prime}(r)$ ), a contradiction to the maximality for the circles; see Fig. 7(b)

Using the lemma, we can obtain a quadratic-time algorithm as follows. Given a subgraph $G$ of a fan, compute the balanced circle-contact representation $\Gamma$ for the corresponding star $T$ using Draw_Tree. Then pick the vertices of $T$ other than the center in an arbitrary order and for each vertex $v$, replace the circle $c(v)$ in $\Gamma$ by a circle of maximum size that does not violate any of the properties $(\mathrm{P} 1)-(\mathrm{P} 4)$ in the proof of Lemma 3 This takes a linear time. Now for every edge $(x, y)$ for which $c(x)$ and $c(y)$ do not touch, replace one of the two circles (say, $c(y)$ ) with a circle that touches $c(x)$ as in Fig. 7(b) Note that this may result in a loss of a contact between $c(y)$ and the circle to its right. We perform a similar operation for the circle to the right of $c(y)$, then possibly for the circle on its right and so on, until all missing contact are repaired. This process requires linear time per edge; hence, the total running time to compute the desired contact representation is quadratic. The contact representation is balanced since the representation obtained by Draw_Tree is balanced and afterwards we only increase the size of circles that are not of the largest size.

Theorem 3. Every n-vertex fan-tree graph has a balanced circle-contact representation. Such a representation can be found in $\mathcal{O}\left(n^{2}\right)$ time.

Proof: Let $G$ be a fan-tree graph and let $T$ be the corresponding tree for which $G$ is the augmented fan-tree. Using Draw_Tree, we first obtain a balanced circle-contact representation of $T$. As in the proof of Lemma 3 this defines a vertical strip for each vertex in $T$. In a top-down traversal of $T$, we can find a contact representation of $G$ with circles by repeating the quadratic-time algorithm for the subgraphs of fans. Hence, the total complexity is $\sum_{v \in V(T)} \operatorname{deg}_{T}^{2}(v)=\mathcal{O}\left(n^{2}\right)$.

As a corollary of Theorem 3 , we obtain an algorithm for creating balanced circlecontact representation of a cactus graph.

Corollary 1. Every n-vertex cactus graph has a balanced circle-contact representation. Such a representation can be found in $\mathcal{O}\left(n^{2}\right)$ time. 


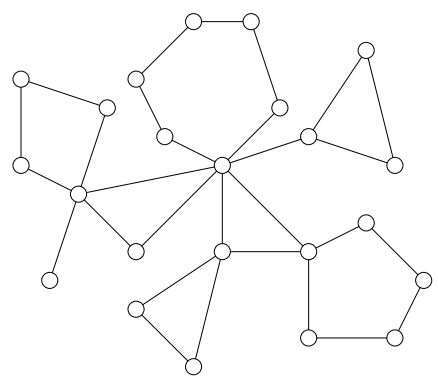

(a)

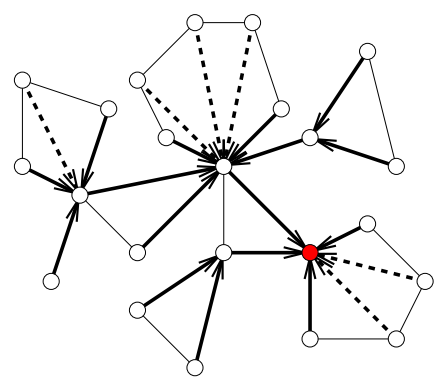

(b)

Fig. 8. (a) A cactus graph $G$; (b) augmenting $G$ to a fan-tree so that the directed edges form a rooted tree and are oriented towards the root

Proof: Given cactus graph $G$, choose a root vertex $v$ arbitrarily. For each cycle $C$ of $G$, add an edge from each vertex of $C$ to the (unique) closest vertex to $v$ in $C$ (Fig. 8). The resulting supergraph of $G$ is a fan-tree; the result follows by Theorem 3.

In the full version of this paper [1], we provide a linear-time algorithm for balanced circle-contact representation of outerpaths. The main idea of this construction is to partition a given outerpath into a sequence of fans, use unit circles to represent the zigzag outerpath formed by the vertices at the ends of each fan, and then perturb these circles by small rotations to make room for the other circles that should go between them.

Theorem 4. Every outerpath has a balanced circle-contact representation. Such a representation can be found in linear time.

\section{Bounded Tree-Depth}

A graph $G$ has tree-depth $t$ if there exists a supergraph of $G$, and a depth-first search tree $T$ of the supergraph, with at most $t$ vertices on every root-leaf path in $T$. A family of graphs has bounded tree-depth if and only if there is a constant bound on the length of the longest path that can be found in any of its graphs [20].

Theorem 5. For every constant bound d, every planar graph with tree-depth at most $d$ has a balanced circle-contact representation.

We sketch the proof from the full version of this paper [1]. The first step characterizes the planar graphs with bounded tree-depth, using block-cut trees and SPQR trees to represent the 2-vertex-connected and 3-vertex-connected components of a graph. We show that a family of planar graphs has bounded tree-depth if and only if the blockcut trees of graphs in the family have bounded depth, the SPQR trees of 2-connected components of these graphs have bounded depth, and each 3-connected component has a bounded number of vertices. If all three conditions are true, the longest path length can be bounded by a recursion of bounded height and branching factor. Conversely, if any one of these conditions is violated, then there exist paths of unbounded length: a long 
path in one of the trees leads directly to a long path in the graph and large 3-connected components have long paths by results of Chen and $\mathrm{Yu}$ [6].

Because each 3-connected component must have bounded size, the circle packing theorem gives it a balanced circle packing. Next, we construct a contact representation for a supergraph of the given graph, by using Möbius transformations to glue together these packings. The virtual edge representing two adjacent components in an SPQR tree should be represented by a pair of tangent circles shared by the packings for the two components; two tangent circles may be shared by an unbounded number of components. We find a family of Möbius transformations that pack all these components into the space surrounding the two shared tangent circles, so that the components are otherwise disjoint from each other, and each is distorted by a polynomial factor. By using this method to combine adjacent nodes of the block-cut and SPQR trees, we obtain a balanced circle packing for the whole graph in which each component is transformed a constant number of times with polynomial distortion per transformation. However, we may have additional unwanted tangencies between circles, coming from virtual edges in an SPQR tree node that do not correspond to graph edges.

The final part of our proof of Theorem 5 shows how to perturb these glued-together packings, in a controlled way, to eliminate the contacts between pairs of vertices that are connected by virtual edges but not by edges of the input graph while still allowing the Möbius gluing to work correctly. The existence of a Möbius transformation from one pair of circles to another is controlled by an invariant of pairs of circles called their inversive distance that equals 1 for tangent circles, is less than 1 for crossing circles, and is greater than 1 for disjoint circles. The theory of inversive distance circle packings is not as well-developed as the theory of tangent circle packings, but a theorem of Luo [17] implies that, for a maximal planar graph with specified positions for the centers of the three circles representing the outer face of the graph and specified inversive distances on each edge of the graph, a circle packing of this type is unique when it exists. By combining this fact with Brouwer's theorem of invariance of domain, we show that for any fixed maximal planar graph (and fixed three outer circle centers) the space of feasible assignments of inversive distances to edges of the graph forms an open set. Therefore, for all sufficiently small $\varepsilon>0$, there exist packings for which all virtualbut-not-actual edges have inversive distance $1+\varepsilon$ and all actual edges have inversive distance 1 . Choosing $\varepsilon$ to be inverse-polynomially small allows the same gluing method to complete the construction and the proof.

\section{Conclusion}

We studied balanced circle packings for planar graphs, showing that several rich classes of graphs have balanced circle packings. One interesting open problem is whether or not every outerplanar graph has a balanced circle packing representation. While we identified several subclasses of outerplanar graphs that admit such representations, the question remains open for general outerplanar graphs.

Acknowledgments. This work is supported in part by the National Science Foundation under grants CCF-1228639, CCF-1115971, DEB 1053573, and by the Office of Naval Research under Grant No. N00014-08-1-1015. 


\section{References}

1. Alam, J., Eppstein, D., Goodrich, M.T., Kobourov, S.G., Pupyrev, S.: Balanced circle packings for planar graphs. Arxiv report arxiv.org/abs/1408.4902 (2014)

2. Bannister, M.J., Devanny, W.E., Eppstein, D., Goodrich, M.T.: The Galois complexity of graph drawing: Why numerical solutions are ubiquitous for force-directed, spectral, and circle packing drawings. In: Duncan, C., Symvonis, A. (eds.) GD 2014. LNCS, vol. 8871, pp. 149-161. Springer, Heidelberg (2014)

3. Bern, M., Eppstein, D.: Optimal Möbius transformations for information visualization and meshing. In: Dehne, F., Sack, J.-R., Tamassia, R. (eds.) WADS 2001. LNCS, vol. 2125, pp. 14-25. Springer, Heidelberg (2001)

4. Breu, H., Kirkpatrick, D.G.: Unit disk graph recognition is NP-hard. Comput. Geom. Th. Appl. 9(1-2), 3-24 (1998)

5. Brightwell, G., Scheinerman, E.: Representations of planar graphs. SIAM J. Discrete Math. 6(2), 214-229 (1993)

6. Chen, G., Yu, X.: Long cycles in 3-connected graphs. J. Comb. Theory B 86(1), 80-99 (2002)

7. Collins, C.R., Stephenson, K.: A circle packing algorithm. Comput. Geom. Th. Appl. 25(3), 233-256 (2003)

8. Dolev, D., Leighton, T., Trickey, H.: Planar embedding of planar graphs. Advances in Computing Research 2, 147-161 (1984)

9. Duncan, C.A., Gansner, E.R., Hu, Y.F., Kaufmann, M., Kobourov, S.G.: Optimal polygonal representation of planar graphs. Algorithmica 63(3), 672-691 (2012)

10. Eppstein, D.: Planar Lombardi drawings for subcubic graphs. In: Didimo, W., Patrignani, M. (eds.) GD 2012. LNCS, vol. 7704, pp. 126-137. Springer, Heidelberg (2013)

11. Eppstein, D., Holten, D., Löffler, M., Nöllenburg, M., Speckmann, B., Verbeek, K.: Strict confluent drawing. In: Wismath, S., Wolff, A. (eds.) GD 2013. LNCS, vol. 8242, pp. 352363. Springer, Heidelberg (2013)

12. de Fraysseix, H., de Mendez, P.O., Rosenstiehl, P.: On triangle contact graphs. Combinatorics, Probability \& Computing 3(2), 233-246 (1994)

13. Gilbert, E.N., Moore, E.F.: Variable-length binary encodings. Bell System Technical Journal 38(4), 933-967 (1959)

14. Gonçalves, D., Lévêque, B., Pinlou, A.: Triangle contact representations and duality. Discrete Comput. Geom. 48(1), 239-254 (2012)

15. Hliněný, P.: Classes and recognition of curve contact graphs. J. Comb. Theory B 74(1), 87103 (1998)

16. Koebe, P.: Kontaktprobleme der konformen Abbildung. Ber. Sächs. Akad. Wiss. Leipzig, Math.-Phys. K1. 88, 141-164 (1936)

17. Luo, F.: Rigidity of polyhedral surfaces, III. Geometry \& Topology 15(4), 2299-2319 (2011)

18. Malitz, S.M., Papakostas, A.: On the angular resolution of planar graphs. SIAM J. Discrete Math. 7(2), 172-183 (1994)

19. Mohar, B.: A polynomial time circle packing algorithm. Discrete Math. 117(1-3), 257-263 (1993)

20. Nešetřil, J., Ossona de Mendez, P.: Sparsity: Graphs, Structures, and Algorithms. Springer (2012)

21. Nievergelt, J., Reingold, E.M.: Binary search trees of bounded balance. SIAM J. Comput. 2, 33-43 (1973) 